\title{
UNA ALTERNATIVA PARA EL ESCEPTICISMO INTERPRETATIVO: CONVENCIONES Y CUASIRREALISMO EN LA INTERPRETACIÓN JURÍDICA*
}

\author{
Federico José Arena \\ Università Bocconi
}

RESUMEN. En este trabajo el autor analiza algunas dificultades que, desde su punto de vista, acechan al escepticismo interpretativo. Según el autor, esta posición se enfrenta a dos dilemas, uno interno y otro externo. Ambos dilemas se producen cuando las tesis escépticas son comparadas con ciertas afirmaciones que los jueces hacen respecto de los enunciados interpretativos y las normas jurídicas. El autor propone una estrategia convencionalista, que recurriendo a la noción de convenciones interpretativas, permite escapar del dilema interno; mientras que el dilema externo puede ser desarticulado mediante una estrategia cuasirrealista, de acuerdo a la cual, la posibilidad de predicar la verdad de ciertos enunciados no depende de la existencia de las entidades a las que tales enunciados se refieren. Ambas estrategias exigen abandonar la tesis escéptica según la cual los enunciados interpretativos carecen de valor de verdad, pero el autor sostiene que se trata de una herida aparente y que el escepticismo sale finalmente ileso.

Palabras clave: enunciado interpretativo, enunciado de deber, constraint hermenéutico, teoría del error.

\section{An Alternative to Rule Skepticism: Conventions and Quasi-realism in Legal Interpretation}

ABSTRACT. The author analyzes some pitfalls that threaten Rule Skepticism. According to the author, Rule Skepticism faces an internal dilemma and an external dilemma. Both dilemmas rise when the theses defended by skepticism are considered in relation with some claims judges make regarding interpretative statements and legal norms. The author proposes, first, that skepticism can get out of the internal dilemma by adopting a conventionalist strategy, i. e. a strategy that resort to interpretative conventions. Second, that in order to dismantle the external dilemma, skepticism should adopt a quasi-realist strategy, i. e. a strategy according to which for a statement to be true, it is not necessary that the object to which it makes reference exists. Both strategies requires skepticism to give up the thesis that interpretative statements have no truth value, but the author claims that it is just an apparent damage and that Rule Skepticism remains unharmed. theory.

Keywords: interpretative statement, statement of duty, hermeneutic constraint, error

* Fecha de recepción: 16 de julio de 2013. Fecha de aceptación: 20 de septiembre de 2013.

Son muchas las personas que me han ayudado con sus críticas y observaciones. Quisiera agradecer en particular a D. CANALE, J. FerRer, R. Guastini, L. RAmíreZ y P. RAPetTi. 


\section{INTRODUCCIÓN}

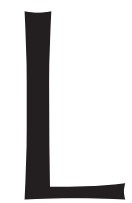

os filósofos del derecho suelen discutir sobre el valor de verdad de los enunciados a través de los cuales los jueces identifican el derecho. Esta preocupación se traduce, por lo general, en la cuestión referida a la posibilidad de predicar corrección o incorrección respecto de tales enunciados. El escepticismo interpretativo es el punto de vista según el cual, por un lado, las disposiciones normativas poseen más de un significado y, por otro lado, los enunciados interpretativos carecen de valor de verdad, por lo que toda afirmación acerca de la corrección o incorrección de estos últimos es una afirmación ideológica ${ }^{1}$.

En este trabajo quisiera analizar algunas dificultades que creo acechan al escepticismo interpretativo. Más específicamente, creo que esta posición enfrenta dos dilemas. El primer dilema se presenta porque no resulta sencillo defender conjuntamente las dos tesis que caracterizan al escepticismo. Como intentaré mostrar, el escéptico debe, o bien afirmar que su posición es independiente de lo que hacen y dicen los jueces, pero en ese caso tiene que abandonar la tesis según la cual los enunciados interpretativos carecen de valor de verdad y termina comprometido con una teoría del error. O bien afirmar que su tesis depende de lo que hacen y dicen los jueces, pero en ese caso parece una tesis falsa respecto de lo que efectivamente hacen y dicen los jueces, ya que éstos usan los enunciados interpretativos del mismo modo en que suelen usarse los enunciados descriptivos, i. e. los enunciados que poseen valor de verdad.

Creo que una estrategia convencionalista, $i$. e. una estrategia que recurre a la noción de convenciones interpretativas, puede solucionar este primer dilema, en cuanto permite dar cuenta del uso que los jueces hacen de los enunciados interpretativos. Según el convencionalismo, detrás del discurso descriptivo de los jueces existe una referencia a convenciones interpretativas que fijan la corrección o incorrección de los enunciados interpretativos.

Sin embargo, sobre la base del resultado de los enunciados interpretativos, los jueces afirman que ciertas personas poseen deberes. Estos enunciados no pueden basarse en la existencia de una convención interpretativa, puesto que la capacidad práctica de las convenciones no es de este tipo. Surge así un segundo dilema que, desde mi punto de vista, es externo ya que depende de introducir consideraciones metaéticas. Según este dilema, el escéptico debe, o bien sostener que la verdad de esos enunciados de los jueces depende de que se correspondan con hechos normativos, pero en ese caso no puede defender una tesis metaética según la cual tales hechos no existen; o bien defender la tesis metaética en cuestión, pero en ese caso queda comprometido con una teoría del error. Argumentaré que este dilema puede ser resuelto adoptando una estrategia cuasirrealista, $i$. e., una estrategia según la cual la posibilidad de predicar verdad de ciertos enunciados no depende de la existencia de las entidades, a las que

1 Si bien para la presentación del escepticismo me basaré aquí en la versión delineada por R. GUASTINI en «El escepticismo ante las reglas replanteado» (GUASTINI, 2012 y GUASTINI, 2011), no estoy seguro que GUASTINI acepte mi reconstrucción de su posición. No sólo porque apelaré, en ocasiones, a otros autores que han sostenido posiciones parecidas a la suya; sino también porque exploraré posibilidades teóricas que, a mi entender, están abiertas para el escéptico pero que no han sido explícitamente defendidas por GUASTINI. 
tales enunciados se refieren. Como veremos, resolver ambos dilemas exige finalmente abandonar la tesis que los enunciados interpretativos carecen de valor de verdad, pero creo que esto es sólo una herida aparente y que, por tanto, el escepticismo sale finalmente ileso.

Para presentar estas ideas dividiré el trabajo en cinco partes. En la primera presentaré el escepticismo interpretativo y en la segunda introduciré el dilema interno. En la tercera parte presentaré la estrategia convencionalista y el modo en el que permite escapar de ese dilema. En la cuarta parte, me referiré al dilema externo y finalmente, en la quinta parte, intentaré avanzar una propuesta cuasirrealista para desarticular este último dilema.

\section{EL ESCEPTICISMO INTERPRETATIVO}

Gran parte de los filósofos del derecho reservaban para el escepticismo interpretativo, al menos en ámbito jurídico, el rincón en el que terminan las teorías que se consideran superadas. Si entendido como la tesis según la cual, el contenido del derecho no es otro más que el que le dan los jueces, el escepticismo interpretativo (de ahora en adelante simplemente «escepticismo») era visto como implausible ${ }^{2}$.

Para defender al escepticismo de algunas de estas críticas GUASTINI propone un replanteamiento. En esta propuesta, GUASTINI traduce la tesis central del escepticismo en una tesis sobre la interpretación ${ }^{3}$. Por interpretación se entiende aquí la actividad que consiste en identificar el derecho, es decir, en determinar el significado de los enunciados de las fuentes ${ }^{4}$, i. e. de los textos normativos. El resultado de esta actividad consiste en (la formulación de) un enunciado interpretativo. Bajo la versión de GUASTINI, la tesis escéptica afirma que los enunciados interpretativos no poseen valor de verdad.

Un enunciado interpretativo es un enunciado de la forma «D significa $N »$, donde $D$ está por el texto normativo (la disposición) y $N$ por su significado (la norma) ${ }^{5}$. El fundamento principal de la tesis escéptica, según GUASTINI, es que los significados, que pueden en abstracto ser atribuidos a una disposición, son por lo general numerosos (o, al menos, más de uno). El hecho que exista esta pluralidad de significados produce indeterminación en el ordenamiento jurídico, puesto que no es posible establecer cuáles son las normas que lo integran.

Siempre según el escéptico, este rasgo del significado de los textos jurídicos depende menos del carácter «objetivamente» equívoco del lenguaje que de otras fuentes como:

${ }^{2}$ En particular sobre la base de las conocidas críticas que HART dirigió al escepticismo en el capítulo 7 [«Formalismo y escepticismo ante las reglas», HART, 1994 (1961)]. Críticas que también A. Ross, primero dirigió contra J. C. GRAY y J. FrANK [en ROSS, 1946 (1934): 62], y posteriormente, intentó responder en RosS, 1958: 36-37.

${ }^{3}$ Las razones para traducir de este modo las tesis escépticas son numerosas. Una de ellas es que en esa actividad se reflejan claramente los estrechos vínculos entre jueces y juristas. Vid., por ejemplo, GUASTINI, 2012: 45 .

${ }^{4}$ Guastini, 2012: 27, y Guastini, 2011: 7-8 y 13-15.

5 GUASTINI, 2012: 45. 
a) la multiplicidad de métodos interpretativos;

b) la dogmática jurídica, y

c) el sentimiento de justicia de los intérpretes, i. e., sus preferencias éticas y políticas ${ }^{6}$.

Dado que los textos jurídicos no poseen un único significado, que pueda ser descubierto, es precisamente a través de la interpretación que los intérpretes deciden, entre una pluralidad de significados, cuál atribuir a la disposición.

En palabras de GUASTINI:

El escepticismo ante las reglas, tal como lo concibo, equivale a sostener que cualquier tesis sobre la existencia de una única interpretación correcta — cualquier tesis que sostenga que los problemas interpretativos admiten una única respuesta «correcta» (i. e. verdadera)_ es falsa. Y es falsa precisamente debido a la indeterminación de sistema jurídico en el sentido especificado, lo que trae como consecuencia que los enunciados interpretativos no tienen valor de verdad. La indeterminación interpretativa del sistema jurídico es el verdadero fundamento del escepticismo ante las reglas (GUASTINI, 2012: 38).

Para advertir cuáles son las implicancias de estas tesis escépticas, me parece necesario comprender en qué consiste la indeterminación cuando se presenta. En los casos de indeterminación, según el escéptico, el único enunciado interpretativo con condiciones de verdad (EIC) que puede formular el intérprete es el siguiente:

$$
\text { (EIC) } D \text { significa } N 1, N 2, N 3 \ldots N n
$$

Este enunciado se limita a afirmar cuáles son los significados a los que, de hecho, un mismo texto resulta asociado. Un enunciado de este tipo es verdadero si, y sólo si, contiene un elenco (completo) de las interpretaciones existentes ${ }^{7}$. Sin embargo, el problema consiste en que un enunciado como (EIC) no permite al intérprete justificar su decisión acerca de qué se debe hacer en un caso concreto, ya que para ello es necesario que identifique o decida cuál es el (único) significado de $D$. Dicho de otro modo, si la solución de un caso depende de cuál es el significado de $D$, entonces, para justificar su decisión, no es suficiente que el intérprete se limite a constatar la existencia de una multiplicidad de significados atribuidos al mismo texto normativo $D^{8}$.

En definitiva, según el escéptico, dado que cada texto normativo de hecho es, y puede ser, interpretado en numerosas maneras diversas, no tiene sentido decir que un intérprete se ha equivocado en atribuir al texto un significado (falso), en lugar de otro significado (verdadero). Esto implica que no existen criterios de verdad (objetivos) que permitan predicar error respecto de los enunciados interpretativos y, por tanto, que toda afirmación de este tipo no es más que una expresión de la ideología o actitudes valorativas del intérprete.

Sobre esta base entenderé por escepticismo una posición que defienda las siguientes tesis:

6 Vid. GUASTINI, 2012: 40, y con algunas variaciones, GUASTINI, 2011: 47-52.

7 Resultado en el que en general se considera termina la tarea cognitiva del intérprete. Vid. GuAstinI, 2011: 27-31, y GUASTINI, 2012: 31-33.

${ }_{8}^{8}$ En el caso de los jueces, sin la atribución de un único significado la decisión judicial no se encontraría justificada, pues las premisas no serían suficientes para llegar a una conclusión. 


\section{Escepticismo interpretativo}

i) Tesis acerca del significado de los textos normativos: los textos normativos poseen una pluralidad de significados como consecuencia de: a) la multiplicidad de métodos interpretativos; $b$ ) la dogmática jurídica, y $c$ ) el sentimiento de justicia de los intérpretes (Tesis de la pluralidad de significados y de determinantes del significado).

ii) Tesis acerca del valor de verdad de los enunciados interpretativos: los enunciados interpretativos carecen siempre de valor de verdad (Tesis de la falta de valor de verdad) ${ }^{9}$.

Desde mi punto de vista, este conjunto de tesis se enfrenta a dos dilemas. Un primer dilema que es interno, en el sentido que su producción depende sólo de asumir las tesis escépticas, y un segundo dilema que es externo, en el sentido que su producción depende de agregar una tesis al conjunto de tesis escépticas. Ambos dilemas surgen de considerar con algo de caridad una de las críticas más insistentes contra el escepticismo. Esta crítica señala que el escepticismo no da cuenta, o no coincide, con ciertas afirmaciones que los jueces parecen compartir respecto de los enunciados interpretativos y las normas jurídicas. En primer lugar, la afirmación según la cual los enunciados interpretativos poseen valor de verdad, o, al menos, es posible predicar (objetivamente) corrección o incorrección respecto de ellos. En segundo lugar, la afirmación según la cual las normas, asociadas a la disposición mediante los enunciados interpretativos correctos, imponen deberes.

\section{EL DILEMA INTERNO}

Como vimos, según la Tesis (ii) del escepticismo, tal como es traducido por GUASTINI, los enunciados interpretativos no poseen valor de verdad. Ahora bien, ¿por qué los enunciados interpretativos carecen de valor de verdad? Creo que a esta pregunta pueden darse dos tipos de respuestas distintas. Por un lado, una respuesta semántica y, por otro lado, una respuesta pragmática ${ }^{10}$.

La primera respuesta, semántica, podría asumir dos versiones. Según una versión (a.1) los enunciados interpretativos carecen de valor de verdad porque las entidades a

9 Las Tesis (i) y (ii) son ciertamente criticadas por otras teorías de la interpretación. En este sentido, las distintas posiciones podrían ser distinguidas sobre la base del contenido que dan a las dos tesis. 1) Formalismo: i) los textos normativos poseen un único significado objetivo, y ii) los enunciados interpretativos poseen siempre valor de verdad. 2) Eclecticismo: i) los textos normativos poseen un significado objetivo que permite la existencia de casos fáciles, pero lamentablemente ese significado es indeterminado, dando lugar a casos difíciles o marginales, y ii) en los casos fáciles los enunciados interpretativos poseen valor de verdad, en los casos difíciles los enunciados interpretativos no poseen valor de verdad. 3) Escepticismo radical: i) los textos normativos no poseen ningún significado, y ii) los enunciados interpretativos carecen siempre de valor de verdad. Es fácil notar que entre el escepticismo radical y el que reconstruyo hay sólo una discrepancia sobre la tesis (i). Para la versión extrema del escepticismo, vid. TROPER, 2001: 74. Como adelanté, aquí me limito a analizar la plausibilidad del escepticismo.

10 Entiendo esta distinción meramente como aquella entre ofrecer una respuesta que depende de que existan o no las entidades a las que se refiere el enunciado (semántica) y ofrecer una respuesta que depende de la acción llevada a cabo por quien lo pronuncia o formula (pragmática). 
las que hacen referencia no existen. Sin embargo, claramente esta tesis no está disponible para el escéptico.

En primer lugar, porque el escéptico acepta efectivamente que los significados existen. Enumerar los significados que son, y pueden ser, asociados a una disposición -i. e., formular enunciados del tipo (EIC) - , es una actividad que goza legítimamente, según el escéptico, de la etiqueta de actividad interpretativa cuyo resultado es un enunciado verdadero o falso.

En segundo lugar, porque no sería de todos modos una buena respuesta. Por ejemplo: «El rey de Francia es calvo». ¿Es un enunciado falso o un enunciado que no posee valor de verdad? Según Russell, la estructura lógica de ese enunciado revela que se trata de una conjunción de dos enunciados: «Existe un $x$ tal que $x$ es rey de Francia y $X$ es calvo». Así, dado que el rey de Francia no existe, se trata de un enunciado falso, puesto que uno de sus elementos es falso ${ }^{11}$. Del mismo modo podríamos preguntarnos, ¿el enunciado interpretativo $\langle D$ significa $N »$, es falso o carece de valor de verdad? Si aceptamos el análisis de RuSSELL, la estructura lógica del enunciado sería «Existe un $x$ tal que $x$ es el significado $N$ y $x$ es el significado de $D »$. En ese caso, si los significados no existen, entonces enunciados de ese tipo serían sistemáticamente falsos, pero ciertamente no carecerían de valor de verdad.

Según otra versión (a.2) los enunciados interpretativos carecen de valor de verdad porque asocian a una disposición un único significado, cuando en realidad las disposiciones normativas no poseen un único significado, sino una pluralidad de ellos. Así, los enunciados interpretativos carecen de valor de verdad, no porque la entidad no existe, sino porque las disposiciones no poseen un único significado, sino varios. Bajo esta versión, la Tesis (ii) se basaría en la Tesis (i). Sin embargo, esta respuesta no funcionaría, ya que del hecho que los textos normativos poseen una pluralidad de significados, se sigue que los enunciados interpretativos, en cuanto afirman que tales textos poseen un único significado, son todos falsos ${ }^{12}$. Falsos, pero con valor de verdad.

En conclusión, eligiendo una respuesta semántica, el escepticismo quedaría comprometido con algún tipo de teoría del error y debería aceptar que los enunciados interpretativos poseen valor de verdad. En la versión (a.1) el error recaería sobre la

11 Según RussELl son las variables ligadas o de cuantificación las que nos atan a la existencia de las entidades a las que se refieren, si es que postulamos la verdad de los enunciados que contienen tales variables. De esta manera en la medida en que las variables de cuantificación aparecen en nuestro discurso uno adquiere compromisos con una ontología (RUSSELL, 1905).

12 La tesis según la cual los textos normativos no poseen un único significado es ambigua entre: (i.a) poseen varios significados; (i.b) es indeterminado cuáles significados poseen, y (i.c) no poseen ningún significado. En la definición que he introducido, el escepticismo elige la opción (i.a). Las razones de ello son las siguientes. En primer lugar, el escepticismo debe descartar la versión (i.c.) en cuanto ello implicaría asumir la versión radical del escepticismo. En segundo lugar, la opción (i.b) queda descartada porque, si fuera indeterminado cuáles significados posee el texto normativo, entonces ni siquiera los enunciados interpretativos del tipo (EIC) poseerían valor de verdad. Puesto que ninguna lista de significados puede describir un conjunto indeterminado de significados, al igual que no es posible confeccionar una lista completa de todos los casos que entran en el significado de un término vago. Por ello, o bien los textos normativos poseen varios significados (i.a) y la descripción de (todos) estos significados mediante enunciados del tipo (EIC) es posible, pero entonces afirmar que poseen sólo un significado es una afirmación falsa y por tanto los enunciados interpretativos poseen valor de verdad; o bien cuáles significados posea un texto normativo es indeterminado (i.b) y es posible afirmar, sobre esa base, que los enunciados interpretativos carecen de valor de verdad, pero entonces ni siquiera los enunciados del tipo (EIC) poseen valor de verdad. 
existencia de significados y, en ese caso, se trataría de una teoría del error; análoga a la que algunos sostienen se verifica en ámbito moral donde el error recaería sobre la existencia de hechos morales ${ }^{13}$. Esta versión, además, es incompatible con otras tesis escépticas, en particular con aquella según la cual enunciados como (EIC) poseen valor de verdad y algunos son verdaderos. En la versión (a.2), en cambio, el error no recae sobre la existencia de significados, el error tampoco recae sobre el valor de verdad de los enunciados interpretativos. El error consiste, en cambio, en creer que los textos normativos poseen un único significado.

Si bien los defensores del escepticismo han, de hecho, rechazado abrazar una teoría del error ${ }^{14}$, conviene, creo, explicitar cuáles son las dificultades que pone una teoría de este tipo. Se trata de que, si el uso del lenguaje interpretativo contiene un error, entonces es mejor reemplazarlo por otro uso que evite ese error, ya que resulta sospechoso afirmar que existe un error pero seguir usando del mismo modo un lenguaje. Es por ello que, una primera consecuencia de una teoría del error sería la de impulsar el reemplazo del vocabulario o los conceptos interpretativos equivocados, por otro que evite el error ${ }^{15}$.

La segunda respuesta, pragmática, podría ser: $b$ ) los enunciados interpretativos carecen de valor de verdad porque no son usados para describir, sino para hacer otra cosa, i. e., para adscribir un significado a una disposición. La tesis escéptica así entendida describe (o reconstruye conceptualmente) las acciones, i. e. los actos lingüísticos, llevadas a cabo por los intérpretes. Esta vía parece prometedora, al menos en abstracto. Pero la estrategia pragmática, para ser completa, requiere indicar quiénes son los agentes cuyas acciones se pretende describir o reconstruir y, además, requiere mostrar que el acto lingüístico llevado a cabo por esos agentes es del tipo señalado.

El escéptico afirma, ciertamente, que el conjunto de los enunciados interpretativos a los que se refiere incluye aquellos formulados por los jueces en sus sentencias. Y, agrega, basta con observar lo que hacen los jueces para advertir la corrección de las tesis escépticas. Así, por ejemplo, el escéptico afirma que para advertir que los enunciados interpretativos no son usados para describir «è sufficiente osservare cosa fanno $i$ giuristi - professori di diritto, giudici, avocati- quando interpretano un documento normativo (la costituzione, gli articoli di un codice, un regolamento governativo, ecc.)» ${ }^{16}$.

Entonces, ¿cómo es posible establecer que los jueces usan los enunciados interpretativos para adscribir significado y no para describir el significado de los textos

13 Vid. MackIE, 1977: 30-35.

14 Para GUASTINI, por ejemplo, si se acepta que todos los enunciados interpretativos son falsos, entonces «la pratica interpretativa — che pure è opera non di poveri sprovveduti, ma di giuristi e giudici competenti-darebbe luogo ad una quantità sterminata di enunciati interpretativi falsi. Non sarebbe davvero strano?» (GUASTINI, 2008: 167).

15 Desde este punto de vista, un lenguaje que contenga un error se vería afectado «in something like the way in which phlogiston theory or witchcraft explanations are so tainted. If we come to believe in this error, one response is to abandon the whole thing: to cease to go in for morality, just as we have grown out of phlogiston and witchcraft theories» (BLACKBURN, 2005: 326-327). El defensor de una teoría del error podría, quizá, alegar a su favor que hay consideraciones, externas al modo en que los participantes conciben el hecho social, como por ejemplo la utilidad social de una antigua práctica, que incluso si advertidas por los participantes permitirían la continuidad del hecho social, aun bajo la conciencia del error generalizado.

16 Chiassoni, 1998: 21. 
normativos? $\mathrm{O}$, dicho de otro modo, ¿cómo es posible establecer que los enunciados interpretativos de los jueces son enunciados adscriptivos? Para responder a esta pregunta existen, en principio, dos opciones. Primero, se podría intentar mostrar que es necesario que realicen ese (tipo de) acto. Pero, si aceptamos lo que he apenas dicho acerca de la opción semántica, entonces esta opción queda descartada puesto que es posible que lleven a cabo un acto descriptivo. Segundo, se podría intentar mostrar que se trata del (tipo de) acto o acción que tienen la intención de realizar.

En este sentido, suele afirmarse que la explicación de una acción ha de hacer referencia a las intenciones del agente y, si esa acción forma parte de una práctica, la explicación de la práctica social ha de hacer referencia al modo en que los mismos participantes la conciben. Se trata de una imposición metodológica para quien se embarque en la tarea de describir una práctica y la hipótesis es aquí que la relevancia de la intención (o del punto de vista de los participantes) es una exigencia de la cual una teoría no puede simplemente desentenderse ${ }^{17}$. La formulación que adoptaré es la siguiente:

Constraint Hermenéutico: los hechos sociales han de ser explicados mediante referencia al modo en que los mismos participantes los conciben.

$\mathrm{Ni}$ las intenciones de los agentes, ni el modo en el cual, en cuanto participantes, conciben la práctica se encuentran directamente accesibles al teórico. En consecuencia, la satisfacción del constraint hermenéutico exige un trabajo de reconstrucción, a partir del comportamiento y ciertas respuestas lingüísticas. La reconstrucción así obtenida, debe reflejar una concepción de la práctica que pueda ser asumida por los participantes, en este caso los jueces y las juezas.

La dificultad para el escéptico se presenta porque los jueces usan los enunciados interpretativos del mismo modo en que suelen usarse enunciados descriptivos. Además, los jueces afirman que, al interpretar, encuentran o descubren el significado de los textos normativos. Si ello es así, entonces no sería temerario afirmar que los jueces tienen la intención de formular un enunciado descriptivo. Y no sólo eso, dado que los significados existen, es también posible que algunos enunciados interpretativos sean verdaderos (o correctos). Si ello es así, entonces la Tesis (ii), no sería una buena descripción o reconstrucción del uso de los enunciados interpretativos por parte de los jueces.

Esto pondría en crisis también la Tesis (i), ya que entre los motivos o razones que, según el escepticismo, un juez tiene para formular determinado enunciado interpretativo, se encuentran sus sentimientos de justicia; pero la decisión de adscribir un significado a un término sobre la base de sentimientos de justicia es incompatible con sostener que los enunciados interpretativos son verdaderos o falsos. Si ello es así, entonces «los sentimientos de justicia» no explicarían la proferencia de ciertas afirmaciones por parte de los jueces.

17 Este tipo de preocupación adquirió mayor importancia dentro de la teoría del derecho a partir de la relevancia que el punto de vista interno posee en la obra de HART. Relevancia que parece provenir del hecho que HART comparta las ideas de WINCH [WINCH, $2008(1990,1958)$ y WEBER acerca de la metodología de las ciencias sociales (WEBER, 1968). Pero el argumento que estoy presentando aquí no depende de seguir a HART en el uso que hace de la distinción punto de vista interno/externo. 
Cabe notar además, que siguiendo la opción pragmática no está disponible una teoría del error. Pues, si cuál sea el acto lingüístico llevado a cabo depende de la intención del hablante, entonces el hecho que los hablantes tengan otra intención que la que requeriría la realización de un acto de adscripción del significado, impide sostener que llevan a cabo ese acto. La teoría sería falsa como reconstrucción de lo que hacen los jueces.

Dadas las consecuencias a las que conducen las dos opciones prospectadas se presenta entonces el siguiente dilema interno. $\mathrm{O}$ bien el fundamento del escepticismo es semántico, i. e., las entidades a las que los enunciados hacen referencia no existen (o son numerosas); pero en este caso debe reemplazar la Tesis (ii) por la tesis según la cual los enunciados interpretativos, si bien son todos falsos, poseen valor de verdad, adoptando así una variante de teoría del error. O bien el fundamento del escepticismo es pragmático, i. e., los enunciados interpretativos son usados para atribuir o adscribir significado; pero en este caso, dado que la realización del acto lingüístico que los jueces afirman realizar no es el que señala la Tesis (ii), el escepticismo enfrenta la siguiente dificultad: o bien se compromete con la tesis según la cual el acto que llevan a cabo los jueces no es el que afirman, y tienen la intención de, realizar, pero en ese caso, si bien mantiene la Tesis (ii), no satisface el constraint hermenéutico; o bien satisface el constraint hermenéutico, mostrando que la atribución de significado a disposiciones normativas, sobre la base de los métodos interpretativos existentes en una comunidad jurídica, la dogmática jurídica y los sentimientos de justicia, sirve como explicación de la acción que los jueces afirman, y tienen la intención, de realizar, pero en ese caso, debe abandonar la Tesis (ii).

\section{LA ALTERNATIVA CONVENCIONALISTA PARA EL ESCEPTICISMO}

Una estrategia convencionalista podría, creo, resolver este primer dilema. Como intentaré mostrar, esta estrategia requiere introducir cambios en el conjunto de las tesis escépticas. Por un lado, si bien exige abandonar la Tesis (ii), evita una teoría del error. Y, por otro lado, exige afinar la Tesis (i). Creo que estas variaciones no producen una pérdida irreparable para el escéptico. Esta tesis, en cambio, sale fortalecida luego de llevar adelante la estrategia convencionalista. Sin embargo, como veremos al final de esta sección, ello no resuelve completamente los problemas del escepticismo, ya que se presentará un segundo dilema.

El punto de partida de esta estrategia es que la atribución de significado se produce en el contexto de un conjunto de convenciones, que fijan los significados de las disposiciones normativas.

En ámbito interpretativo, la tesis convencionalista puede ser sintetizada como sigue:

Tesis (iii). El significado de los enunciados interpretativos es determinado por convenciones (Tesis convencionalista).

La defensa de esta versión de la tesis convencionalista exige precisar la noción de convención ${ }^{18}$.

18 Digo versión puesto que la tesis convencionalista no recae necesariamente sobre la interpretación y puede ser formulada de manera más general: «El (o cierto) hecho jurídico fundamental es (o está determinado 


\subsection{Persiguiendo las convenciones}

Los sentidos en que se dice de algo que es una convención o que es convencional tienen diferentes amplitudes. Por un lado, en sentido amplio, quiere decir que depende de los hombres, de sus acciones y creencias, u otras actitudes. En este sentido convencional se opone a natural ${ }^{19}$ y es sinónimo de social ${ }^{20}$. En un sentido más restringido, que es una especificación del anterior, sólo una subclase de los hechos que dependen de las acciones y actitudes humanas son convenciones. Dicho de otro modo, sólo cuando las acciones y actitudes humanas adquieren una cierta configuración nos encontramos frente a una convención. A este conjunto de circunstancias que deben darse para que exista una convención las llamaré condiciones de convencionalidad ${ }^{21}$.

En general se distinguen dos conjuntos de condiciones de convencionalidad. En primer lugar, las condiciones de convencionalidad que definen las convenciones basadas en el acuerdo. Así, si asumimos que un acuerdo consiste en un intercambio de promesas condicionales, por ejemplo, de usar cierto término en un determinado modo, si el otro también promete hacerlo, entonces existe una convención entre los miembros del grupo $\mathrm{G}$, de realizar la acción $A$ en la ocasión $\mathrm{O}$, si cada uno de los miembros de $\mathrm{G}$ ha prometido a los demás hacer $\mathrm{A}$ en $\mathrm{O}$ bajo la condición que los demás prometan hacer $\mathrm{A}$ en $\mathrm{O}^{22}$.

En segundo lugar, el conjunto de condiciones de convencionalidad que definen las convenciones no basadas en el acuerdo ${ }^{23}$. Desde mi punto de vista, la noción de convención basada en el acuerdo es secundaria respecto de aquella no basada en el acuerdo, aunque no podré extenderme aquí sobre esta última afirmación ${ }^{24}$. Lo importante aquí es, en cambio, ofrecer un relato acerca de cómo un hecho puede constituir

por) una convención». Así, la tesis convencionalista tiene dos elementos principales. Por un lado «hecho jurídico» y por otro lado «convención». La elucidación del primer elemento exige precisar el aspecto del derecho respecto del cual se predica su convencionalidad, en nuestro caso, la práctica interpretativa. La elucidación del segundo exige precisar la noción de convención. He abordado con más detalle este análisis en ARENA, 2013.

19 Vid. Celano, 2010 (1997): 281-282, y Vilajosana Rubio, 2010: 139-140.

20 Esta distinción ha de ser matizada pues las acciones y las creencias humanas son parte de lo natural, y en consecuencia, también lo son las convenciones. Vid. KEKES, 1989: 26-27.

${ }^{21}$ Para el análisis de la noción de convención me he apoyado en la literatura central sobre el tema, a saber: LEWIS, 1969; MARMOR, 2009, y CELANO, 2010.

${ }^{22}$ Sigo aquí la concepción estándar, y quizá la más extendida, de acuerdo (LEWIS, 1969: 34; GILBERT, 1993: 627, y RAZ, 1984: 202-203). Las promesas han de ser condicionales pues de otro modo el intercambio no poseería las propiedades que, pre-analíticamente, se atribuye a los acuerdos. Como por ejemplo que no existe acuerdo hasta tanto todos los involucrados no hayan manifestado su voluntad de formar parte de él.

${ }^{23}$ La identificación precisa de estas condiciones se debe, sobre todo, a D. LEWIS. Vid. LEWIS, 1969.

${ }^{24}$ Ello se puede advertir si se analiza la noción de promesa. La versión más extendida de las promesas es la que las concibe como un acto comunicativo, dentro de una práctica de prometer (RAWLS, 1971: 344-350). Según esta concepción, prometer consiste en llevar a cabo ciertos actos comunicativos que, en cuanto satisfacen las condiciones establecidas por las reglas de la práctica convencional de prometer, pueden ser considerados promesas. Es precisamente este modo de concebir las promesas el que introduce el problema de la circularidad cuya solución, creo, exige aceptar que las convenciones basadas en el acuerdo son secundarias respecto de aquellas no basadas en el acuerdo. Es decir, parece claro que el carácter convencional de la práctica del prometer no puede a su vez depender de un acuerdo, puesto que ello produciría una circularidad [una versión alternativa de las promesas, basada en una teoría de las expectativas, es articulada por Th. SCANLON (SCANLON, 1990) y en el capítulo 7 de SCANLON, 1998, pero desde mi punto de vista tampoco resuelve el problema de la circularidad]. 
una convención independientemente de la existencia de un acuerdo. En este sentido, no debe confundirse la tarea de explicar cómo es posible que surja una convención (problema conocido, por los teóricos de los juegos, como el de ontogénesis de las convenciones), y el problema acerca de cuáles son las condiciones de existencia de una convención, independientemente de cómo haya surgido. En lo que sigue se trata de abordar este último problema.

Así como en el caso del acuerdo, la existencia de la convención depende de un comportamiento de los involucrados (por ejemplo, como vimos, el intercambio de promesas condicionales), el primer elemento de una convención no basada en el acuerdo sigue siendo el comportamiento de ciertos individuos.

En este sentido, una primera condición para la existencia de una convención sin acuerdo es que se verifique una cierta regularidad o convergencia de comportamiento. Una regularidad de comportamiento puede ser concebida como «realizar la acción A en la ocasión $\mathrm{O} »$, o puesto de modo más general «A en $\mathrm{O}{ }^{25}$. La regularidad exige que O se presente con cierta frecuencia, o al menos que se haya presentado más de una vez y que sea posible que vuelva a presentarse ${ }^{26}$. Así, existe una regularidad de comportamiento en un grupo $G$, cuando los miembros de $G$ realizan la acción $A$ cada vez que se presenta la ocasión $\mathrm{O}$.

La regularidad o convergencia de comportamiento se encuentra todavía lejos de constituir una convención, se trata claramente de condición necesaria, pero aún no suficiente. Sobre todo porque las convenciones no son hábitos y, siguiendo las clásicas consideraciones de HART, la sola convergencia de comportamiento no es suficiente para llevar a cabo esta distinción ${ }^{27}$.

La segunda condición, que permite pasar de una mera convergencia de conducta a una convención, es una actitud de los involucrados. La más básica de ellas es denominada por la literatura, «condición de dependencia». La condición de dependencia se refiere al contenido de la actitud de quienes forman parte de la convergencia. Cuando se trata de una convención, parece existir un sentido crucial en el cual decimos que nos conformamos a la regularidad, en parte, debido a que otros lo hacen. En particular, la razón del seguimiento está constituida (al menos en parte) por el hecho de que existe una práctica común ${ }^{28}$.

Finalmente, la tercera condición para la existencia de una convención sin acuerdo es la arbitrariedad. La arbitrariedad permite distinguir las convenciones de otros tipos de reglas. Si bien no ha sido fácil precisar en qué consiste, bastará aquí con concebirla como existencia de alternativa ${ }^{29}$. Si una regularidad de comportamiento es un elemento de una convención, entonces ha de existir una regularidad de comportamiento

25 Vid. GILBERT, 2008: 8.

26 GILBERT, 1983: 229.

27 HART, 1994 (1961): 51-61.

28 «Sussiste una convenzione, $R$, quando ciascuno dei membri del gruppo si conforma a $R$ perché cosi fanno gli altri: il fatto che gli altri si conformano a $R$ è, per ciascuno, una ragione (o una delle ragioni principali, ecc.) per conformarsi $>$ [CELANO, 2010 (2003): 330].

29 Por ejemplo, siguiendo a FORSTER, es posible identificar tres sentidos de arbitrariedad: a) Como existencia de una alternativa. b) Como ausencia de justificación. c) Como resultado de un acto de voluntad. Vid. FORSTER, 2004. 
alternativa ${ }^{30}$. Así, la convención según la cual son los padres de la novia quienes han de pagar los gastos de la fiesta de casamiento, bien podría haber sido distinta. Es decir, bien habrían podido ser los padres del novio los encargados de los gastos. La existencia de un modo distinto de configurar una determinada convención puede advertirse claramente en aquellos casos donde ambas posibilidades se dan en el mundo actual, como por ejemplo, el sentido de circulación de los automóviles ${ }^{31}$. Algunas convenciones han adquirido su contenido actual en virtud de cierta circunstancia particular (decisión de una persona o de un grupo, accidente histórico, coincidencia, etc.), pero bien podrían haber sido diferentes y no obstante satisfacer la misma finalidad.

En definitiva, existen dos nociones de convención, una basada en el acuerdo y otra no basada en el acuerdo. Las condiciones de existencia de la primera incluyen el intercambio de promesas condicionales, $i$. e., un acuerdo. Las condiciones de existencia de la segunda consisten en la convergencia de comportamiento, la condición de dependencia y la arbitrariedad ${ }^{32}$.

\subsection{Las convenciones interpretativas}

Existe un sentido evidente según el cual la interpretación depende de convenciones. Las palabras o los enunciados no posean un significado natural o dado, por el contrario, son los individuos o usuarios quienes atribuyen significado a las marcas y a los sonidos. Si esto fuera todo lo que sostiene al respecto la tesis convencionalista, entonces sería una tesis pacífica pero, creo, poco interesante. Además, la posible determinación del significado sobre la base de las convenciones del lenguaje ordinario, no se traslada necesariamente al contexto de la interpretación jurídica ${ }^{33}$. Pero sobre todo, este convencionalismo evidente no daría cuenta de la complejidad de la práctica interpretativa en el ámbito jurídico.

30 La arbitrariedad no ha de ser confundida con la indiferencia. Ni tampoco la arbitrariedad implica indiferencia. El hecho que exista otra regularidad a la que haya sido posible conformarse, no quiere decir que los agentes sean indiferentes (no tengan preferencias) entre esa regularidad y aquella a la que efectivamente se conforman. Vid. LeWIS, 1969: 76-80; MARMOR, 2009: 8-9, y Vilajosana RubIO, 2010: 176.

31 Para ser una alternativa, la regularidad debe satisfacer ciertas condiciones adicionales: a) Practicabilidad: que el mismo grupo pueda conformarse a la regularidad alternativa en las mismas circunstancias. b) Incompatibilidad: que no sea posible para el mismo grupo conformarse a ambas regularidades al mismo tiempo. c) Sin pérdida en el propósito o función. Es decir, que el conformarse a la regularidad alternativa no signifique una pérdida significativa en la función o el propósito que satisface la regularidad actual. Sobre las condiciones para la existencia de alternativa, vid. LEWIS, 1969: 71, y MARMOR, 2009: 10.

32 Existe una propiedad ulterior que ha sido propuesta como condición de convencionalidad, a saber, el conocimiento común. El hecho que $p$ es conocimiento común entre los miembros de un grupo $G$ si, y sólo si, cada uno de los miembros de $G$ sabe que $p$, sabe que cada uno de los miembros de $G$ sabe que $p$, sabe que cada uno de los miembros de $G$ sabe que cada uno de los miembros de $G$ sabe que $p$ y así hasta el infinito. Vid. Gilbert, 1983: 230; Celano, 2010 (1995): 198, y Celano, 2010 (1997): 299. Desde mi punto de vista, el conocimiento común no es una condición de convencionalidad [vid. BURGE, 1975: 250, contra CELANO, 2010 (1995)], pero no entraré en esa discusión aquí. Señalaré sólo que si el conocimiento común fuera una condición, entonces no tendría sentido decir que el convencionalismo se propone desvelar el carácter convencional de algunas prácticas sociales que no son consideradas tales. Esto además parece contradecir el hecho que, como resultado de disputas y discusiones, la convencionalidad de algunas prácticas fue reconocida tardíamente, escondida quizá detrás de prejuicios culturales o religiosos.

33 Acerca de la diferencia entre interpretación en contextos de conversación ordinaria e interpretación jurídica, vid. GUASTINI, 2012: 46-50 y 196-197. 
En este ámbito, se usan convenciones interpretativas que no se limitan a las del lenguaje natural, sino que por lo general son más complejas. El convencionalismo evidente, o bien ignora que existen convenciones interpretativas, o bien piensa que éstas se reducen a la convención simple del significado ordinario, dejando de lado gran parte de las convenciones usadas para la identificación del derecho. Tal como afirma BAYÓN, preocuparse sólo por las convenciones semánticas (que fijan el significado literal u ordinario de las palabras) y descuidar otras convenciones interpretativas constituye una mirada incompleta ${ }^{34}$.

En definitiva, reducir los métodos de interpretación al de la interpretación literal sería equivocado. Por ello, del hecho que los jueces desacuerden acerca del contenido de las convenciones que establecen el significado ordinario de los textos jurídicos, o que al interpretar no sigan una convención ordinaria considerada compartida, no se sigue necesariamente que no exista ninguna convención que determine el significado. Además de las convenciones semánticas del lenguaje ordinario, existe un conjunto de convenciones interpretativas jurídicas que fijan el significado que los intérpretes pueden atribuir a los textos jurídicos. Es por ello que, el significado literal u ordinario de un enunciado jurídico no agota el conjunto de convenciones que determinan el contenido del derecho. Claramente, cuál sea la convención interpretativa usada en cada caso es una cuestión contingente.

El modo en que funcionan estas convenciones puede apreciarse también a través de los métodos interpretativos vigentes en una comunidad jurídica. En este sentido CHIASSONI afirma que un código interpretativo (o código hermenéutico) consiste en un conjunto de directivas hermenéuticas. Estas directivas están estructuradas, a su vez, en diferentes niveles, por lo que encontramos directivas primarias ${ }^{35}$, directivas secundarias ${ }^{36}$ y directivas axiomáticas ${ }^{37}$.

Cada código incluye así un conjunto de reglas que definen un cierto tipo de método interpretativo y establecen cómo usarlo ${ }^{38}$. Estas reglas permiten evaluar, al me-

34 BAYÓn MOHINO, 2002: 63-64.

35 Las directivas primarias «son aquellas directivas que indican a los intérpretes recursos sobre la base de los cuales una disposición puede ser traducida en una o más normas explícitas» (CHIASSONI, 2011: 90). Un ejemplo de directivas de este tipo es el conjunto de directivas que conforma la interpretación lingüística, dentro del cual podemos encontrar la que impone atribuir a una disposición el significado que resulta del uso común de las palabras y de las reglas gramaticales de la lengua natural en la que está formulada.

36 Las directivas secundarias «regulan el uso de las directivas primarias y establecen los criterios para evaluar la corrección [...] de las interpretaciones [...] obtenidas sobre la base de ésas» (CHIASSONI, 2011: 111).

37 Por último, las directivas axiomáticas constituyen «el conjunto (la base) de principios últimos —de axiomas- de un código hermenéutico, y consisten habitualmente en reglas finales, que prescriben qué objetivo (normalmente general) de política del derecho debe ser perseguido al interpretar las disposiciones» (CHIASSONI, 2011: 134, y CHIASSONI, 2011: 89. Vid. también CHIASSONI, 2004: 63).

38 Suelen distinguirse dos tipos de convenciones, a saber, convenciones de coordinación y convenciones constitutivas. Las primeras han sido introducidas por D. LEWIS (LEWIS, 1969). Una convención de coordinación es una regularidad de comportamiento que soluciona un problema de coordinación recurrente. Simplificando, un problema de coordinación se presenta cuando dos o más individuos se enfrentan a más de una posibilidad de acción, algunas de las cuales satisfacen igualmente el interés de cada uno de ellos bajo la condición de que los demás lleven a cabo la misma acción. Las segundas han sido introducidas por MARMOR (MARMOR, 2009). Una convención constitutiva es una regularidad de comportamiento(s) que adquiere(n) su significado a partir de reglas que definen una práctica social y el modo de participar en ella. Si bien creo que en la práctica de identificación del derecho es posible encontrar ambos tipos de convenciones, no me parece aquí 
nos de manera relativa, los resultados interpretativos. Es por ello que puede afirmarse que tales códigos son convenciones constitutivas. Esto permite también dar cuenta del hecho que los jueces, al interpretar un cierto texto en un determinado sentido, no lo hacen por lo general de manera aislada y sobre la base de métodos interpretativos de creación individual. Por el contrario, el hecho que un determinado método sea utilizado por los demás jueces es una razón para usarlo. Ciertamente, cuál sea, de hecho, el contenido de cada código hermenéutico usado en una comunidad jurídica y qué tipos y niveles de directivas incluya, es una cuestión de hecho.

Que todas estas convenciones forman parte de la interpretación jurídica se advierte a partir del hecho que los participantes no se ven a sí mismos como infringiendo el derecho, ni como llevando a cabo argumentos morales, ni teniendo un desacuerdo aparente (como ocurriría si describiéramos el caso diciendo que utilizan la palabra «derecho» en sentidos diferentes), cuando usan otros métodos de interpretación además del literal.

Si esta estrategia convencionalista es exitosa, entonces la Tesis (i) del escepticismo puede ser defendida sin recurrir a la Tesis (ii). Es decir, no es necesario afirmar que los enunciados interpretativos no poseen valor de verdad, para sostener que los métodos interpretativos y las teorías dogmáticas desempeñan un papel relevante en la interpretación. Tanto los métodos interpretativos como las teorías dogmáticas forman parte de las convenciones interpretativas, sobre la base de las cuales los jueces asocian un significado a una disposición. Estas convenciones no sólo establecen cuáles son, y cómo usar, los métodos disponibles para obtener ciertos resultados, sino que a veces pueden también determinar directamente el resultado interpretativo. En ambos casos es posible establecer si el resultado cae o no dentro de la convención, siempre que tal convención exista. Ello en cuanto las convenciones no siempre determinan el significado de toda disposición, como tampoco existe una convención última que desempeñe el papel de criterio último para elegir entre los diferentes métodos interpretativos. Es por eso que, a veces, el enunciado interpretativo se limita sí a identificar el resultado determinado por las convenciones, pero otras veces es consecuencia de elegir un método interpretativo para obtener un determinado resultado.

Para ver cómo el tercer elemento mencionado en la Tesis (i), i. e., los sentimientos de justicia de los intérpretes, desempeñan un papel dentro de esta combinación escepticismo-convencionalismo, es necesario antes advertir la insistente presencia de un segundo dilema.

\section{EL DILEMA EXTERNO}

La combinación escepticismo y convencionalismo puede, tal como he intentado demostrar, resolver el dilema interno que he presentado. Sin embargo, un segundo dilema, externo, se presenta si se considera una cuarta tesis de carácter metaético:

Tesis (iv). No hay hechos morales (Tesis metaética).

necesario introducir esta complicación. Para un punto de vista crítico respecto de la posibilidad de distinguir entre ambos tipos, vid. Vilajosana Rubio, 2010: 163. 
Si bien, de hecho, los defensores del escepticismo suelen defender una tesis metaética de este tipo, no siempre se la incluye en las definiciones de escepticismo ${ }^{39}$; y es por ello que la considero una tesis externa.

Como afirmé, uno de los elementos de las convenciones es la condición de dependencia. Esta condición se verifica cuando, parte del contenido de, la actitud a favor de seguir la regularidad consiste en el hecho que los demás también la siguen. La conformidad a una convención posee la capacidad práctica que poseen los intereses, preferencias o deseos. El convencionalismo afirma que, cuando la práctica de identificación de textos y la interpretación consisten en convenciones, lo que mueve a los jueces a la acción es la condición de dependencia. Una vez que la convergencia se ha producido los agentes tienen una razón para actuar de acuerdo a la convención. Al formular un enunciado interpretativo los jueces siguen una convención interpretativa, y sobre la base de la convención es posible afirmar si el enunciado es verdadero o falso. La estrategia convencionalista podría entonces dar cuenta de estas afirmaciones de los jueces.

Ahora bien, sobre la base de algunos enunciados interpretativos los jueces formulan enunciados de deber. Es decir, afirman que la norma, asociada a la disposición a través del enunciado interpretativo, impone a su destinatario el deber de realizar ciertas acciones. En este sentido, los jueces hablan como si los significados pudieran imponer deberes y como si tales deberes pudieran ser objeto de proposiciones, es decir, como si pudieran ser el contenido de actitudes proposicionales. Este modo de hablar parecería indicar que hablan de deberes del mismo modo como se habla de hechos naturales.

La combinación escepticismo-convencionalismo no puede dar cuenta de estos enunciados. Es decir, estos enunciados no pueden basarse en la existencia de una convención interpretativa, precisamente porque la capacidad práctica de las convenciones no es de este tipo. Si bien el convencionalismo puede apelar a un cierto conjunto de motivos y razones, ese conjunto es limitado y no incluye los deberes. Aun cuando se verifique la condición de dependencia, la existencia de una convención interpretativa no podría dar cuenta de la formulación de enunciados de deber.

¿Lo convertiría este límite en una teoría desechable? Es decir, ¿implicaría esto que la combinación escepticismo-convencionalismo fracasa de todos modos?

Una primera reacción del escepticismo podría consistir simplemente en negar que la teoría de la interpretación deba ofrecer una explicación de cómo las normas, asociadas por el intérprete a cada texto normativo, imponen deberes ${ }^{40}$. Esta respuesta

39 Así, vid. ChIASSONI, 1998, y BARBERIs, 2001, y también el mismo GUASTINI, 2012. A diferencia de estos autores, H. BOUVIER incluye una tesis metaética en la caracterización que hace del escepticismo. Vid. BOUVIER, 2012: 273.

40 No se trataría, por tanto, de un genuino problema. El escéptico podría así adoptar la respuesta que algunos autores dan con relación al problema de la capacidad práctica del derecho. Por ejemplo, BULYGIN, refiriéndose al positivismo jurídico, sostiene que «el problema de la normatividad del derecho, entendido como el de su valor moral, no es un problema jurídico, sino moral y, por tanto, su solución pertenece al campo de la filosofía moral y no al de la filosofía jurídica» (BULYGIN, 2006: 96-97). En definitiva, desde este punto de vista, no dar cuenta de la capacidad práctica del derecho es una consecuencia natural de teorías descriptivas del derecho. Y tanto el positivismo jurídico como el escepticismo son teorías de este tipo. 
ciertamente no convencería a todos. Algunos autores, como por ejemplo SCHIAVELLO, entienden que la pregunta acerca de cómo el derecho impone deberes (i.e. la pregunta por la justificación del derecho) es la pregunta política más importante. Poner entre paréntesis la legitimidad del derecho, sostiene SCHIAVELLO, conlleva el riesgo de favorecer actitudes de asentimiento acrítico y de conformismo respecto de las exigencias que el derecho mismo impone a sus destinatarios ${ }^{41}$.

Críticas de este tipo, sin embargo, mostrarían únicamente que el escepticismo podría ser atacado por defender una tesis políticamente cuestionable, pero no podría ser acusado de inconsistencia teórica.

Sin embargo, el problema se presenta si el escepticismo pretende mantener el constraint hermenéutico y, a su vez, defender la Tesis metaética apenas introducida. Ello en cuanto, si la existencia de una convención interpretativa no es suficiente para afirmar que la norma, contenida en el enunciado interpretativo, impone un deber; entonces, para explicar las afirmaciones de los jueces y satisfacer el constraint hermenéutico, es necesario agregar una explicación adicional. En este caso las alternativas son dos.

La primera alternativa podría ser que la existencia del deber se produce cuando la norma, asociada a la disposición mediante el enunciado interpretativo, es verdadera. En ese caso, sin embargo, sería necesario abandonar la tesis metaética, pues, al menos en principio, una norma es verdadera si coincide con hechos normativos o, dicho de otro modo, la verdad de las normas depende de la existencia de hechos normativos. Además, esta alternativa, si bien daría cuenta de las afirmaciones según la cual las normas imponen deberes, dejaría el gusto amargo de transformar a la interpretación en un mero epifenómeno al respecto. No hay ninguna relación entre interpretación y existencia del deber.

La segunda alternativa podría consistir en afirmar que los jueces se equivocan al creer que la norma, asociada al texto normativo mediante el uso de una convención interpretativa, impone deberes. El error proviene del hecho que una norma impone deberes sólo si es verdadera, $i$. e. sólo si coincide con hechos normativos; pero dado que los hechos normativos no existen, es equivocado sostener que las normas imponen deberes. En este caso, el escepticismo podría conservar la Tesis metaética, pero al precio de sostener, de nuevo, una teoría del error.

Llegamos así al segundo dilema, externo. Dado que la existencia de una convención interpretativa no es suficiente para sostener que la norma, asociada a la disposición, impone un deber; entonces el escepticismo, para satisfacer el constraint hermenéutico, se enfrenta a las siguientes opciones. $\mathrm{O}$ bien afirma que la existencia del deber depende de que la norma, asociada a la disposición, coincida con hechos normativos, i. e. sea verdadera; en este caso da cuenta de las afirmaciones de los jueces, pero debe abandonar la Tesis metaética y, además, transforma la interpretación en un mero epifenómeno respecto de la existencia del deber. O bien, mantiene la Tesis metaética, pero entonces debe defender una teoría del error, es decir, debe afirmar que los jueces se equivocan cuando sostienen que las normas, asociadas a la disposición mediante la interpretación, son verdaderas.

${ }^{41}$ Vid. SCHIAVELLO, 2010: 13-16. 


\section{LA ALTERNATIVA CUASIRREALISTA PARA EL ESCEPTICISMO CONVENCIONALISTA}

Un modo para desarticular este dilema externo consistiría en mostrar que, a pesar de no referirse a algo en el mundo — sobre la base de la Tesis metaética-, los enunciados —en apariencia denotativos o representativos- de deber, formulados por los jueces a partir del resultado de la interpretación, pueden explicarse sin recurrir a una teoría del error.

Creo que ello puede lograrse si se afina la tesis metaética. Esto implica notar que, para usar con sentido enunciados de deber, no es necesario que exista la entidad a la cual supuestamente la norma se refiere, como tampoco es necesario que tal entidad exista para que sea posible predicar verdad o falsedad del enunciado de deber ${ }^{42}$. Para ello basta advertir dos características de estos enunciados que identificaré como Tesis (v):

\section{Tesis (v)}

a) Los enunciados de deber son reflejos proposicionales de actitudes valorativas de los jueces ${ }^{43}$ (Tesis proyectivista).

b) Afirmar que un enunciado de deber es verdadero, es simplemente lo mismo que afirmar que existe el deber. A su vez, afirmar que existe el deber no es otra cosa más que expresar la propia actitud hacia la realización de la acción ${ }^{44}$ (Tesis cuasirrealista).

La primera característica coincide con lo que suele denominarse proyectivismo y permite dar cuenta del tercer elemento que, según la Tesis (i) del escepticismo, determina la atribución de significado a las disposiciones normativas, i. e., los sentimientos de justicia del intérprete. En pocas palabras, desde este punto de vista, nuestras actitudes surgen como respuesta a propiedades del mundo que nos rodea ${ }^{45}$. Así, lo que consideramos sensibilidad moral no es otra cosa más que una función de estímulos/ creencias a respuesta/actitudes. Por ejemplo, si Morena ve a Lucas haciendo uso de drogas y afirma «Eso es equivocado», el proyectivismo afirma que dado un conjunto de hechos, i. e., Lucas llevando a cabo ciertas acciones, y la percepción por parte de Morena de esos hechos a partir de las cuales forma ciertas creencias (acerca de lo que está haciendo Lucas, de sus intenciones, de los efectos de lo que hace), se produce una emoción en Morena: de desaprobación frente a lo que hace Lucas. Morena proyecta esta emoción sobre el mundo y a ello sigue su afirmación de que «Eso es equivocado». En virtud de ello, Morena «judges the world to contain a certain quality, and her doing

${ }^{42}$ «The problem is not with a subjective source for value in itself, but with people's inability to come to terms with it, and their consequent need for a picture in which values imprint themselves on a pure passive, receptive witness, who has no responsibility in the matter. To show that these fears have no intellectual justification means developing a concept of moral truth out of the materials to hand: seeing how, given attitudes, given constraints upon them, given a notion of improvement and of possible fault in any sensibility including own, we can construct a notion of truth» (BLACKBURN, 1984: 198).

43 BlaCKBURN, 1998: 77.

44 BlackBURN, 1998: 77-83. Para una presentación de los puntos de vista de BlackBuRn, vid. BOUVIER, 2012.

45 Vid. Blackburn, 1998: 4. 
so is not the product of her tracking a real feature of the world, but is, rather, prompted by an emotional experience» ${ }^{46}$. Así, involucrarse en un discurso sobre deberes consiste en usar el lenguaje para proyectar actitudes ${ }^{47}$.

La segunda característica implica que, cuando un juez profiere un enunciado de deber, a partir del resultado de un enunciado interpretativo, está expresando una actitud (voicing certain attitude) ${ }^{48}$. Lo que hacemos cuando decimos que algo es bueno, o correcto u obligatorio es expresar (avow) nuestro punto de vista práctico ${ }^{49}$. Decir que hay un valor o un deber es desarrollar una cierta actitud hacia cierto estado de cosas que implica estar motivado a actuar en consecuencia ${ }^{50}$.

¿En qué sentido puede usarse el término verdadero respecto de enunciados que expresan una actitud? Siguiendo a BLACKBURN, afirmar que existe un deber implica sostener que se trata de una actitud que pertenece al conjunto de actitudes que han superado todos los test de mejoramiento, o al menos que consideramos imposible de revisar. En palabras de BLACKBURN:

The simple suggestion is that we define a «best possible set of attitudes», thought of as the limiting set which would result from taking all possible opportunities for improvement of attitude. Saying that an evaluative remark is true would be saying that it is a member of such a set, or is implied by such a set. Call the set $\mathrm{M}^{*}$, Then if $m$ is a particular commitment, expressing an attitude $\mathrm{m}$ :

$\mathrm{m}$ is true $=\mathrm{m}$ is a member of $\mathrm{M} *$ (BLACKBURN, 1984: 198).

Sobre esta base, el discurso de los jueces puede ser mantenido aun bajo un esquema proyectivista y cuasirrealista. Ya que, si el cuasirrealismo es exitoso, entonces quiere decir que logra acomodar la gramática proposicional de ese discurso.

Es más, logra mostrar también que es posible hablar de deberes impuestos por el derecho, sin caer en ninguno de los errores que afligen los puntos de vista que afirman la existencia de deberes a partir de hechos sociales. Un juez puede sostener que el derecho impone el deber, por ejemplo, de condenar a diez años de prisión a quien ha causado la muerte de otro, sin necesidad de pensar que tal deber se sigue como consecuencia del hecho que un grupo de individuos ha sancionado un texto al cual puede atribuirse ese significado. Al afirmar esto, el juez está expresando su actitud, su punto de vista o, tal vez, su deseo; y esta expresión permite identificar cómo debería

46 JOYCE, 2007. Vid. BlaCKBuRN, 1998: 4-8.

47 «We project an attitude or habit or other commitment which is not descriptive onto de world, when we speak and think as though there were a property of things which our sayings describe which we can reason about, know about, be wrong about, and so on» (BLACKBURN, 1984: 170-171).

48 Sin embargo, «[t]o bold a value, then, is typically to have a relative stable disposition to conduct practical life and practical discussion in a particular way: it is to be disposed or set in that way, and notably to be set against change in this respect. This way of being set is such as to align values and motivations» (BLACKBURN, 1998: 67).

${ }^{49}$ " "Avowal" here means that we express this state, make it public, or communicate it» (BLACKBURN, 1998: 68).

50 Lo mismo podría afirmarse respecto de lo que a primera vista parece expresar una afirmación metafísica de segundo orden respecto de la moral. Por ejemplo: «Incluso si lo deseáramos o disfrutáramos haciéndolo, el uso de drogas estaría igualmente mal». Esta afirmación parecería ser de segundo orden, y relativa a la independencia entre corrección moral y nuestros deseos, pero un cuasirrealista podría verla como un compromiso de primer orden según el cual no son nuestros deseos los que determinan si está bien o mal usar drogas, sino, principalmente, los efectos que ello produce en las personas. En definitiva, se trata de afirmaciones que expresan en realidad una actitud o necesidad de primer orden. 
cambiar el mundo para que se ajuste a la actitud del juez y qué actitud deberían tomar los demás funcionarios para coincidir con el juez.

Ahora bien, BLACKBURN se refiere a dos tipos de cuasirrealismo, a saber, Slow-track y Fast-Track ${ }^{51}$.

Para la versión slow-track determinar cómo el proyectivismo puede ayudarnos a entender que un enunciado mediante el cual se expresa una actitud pueda funcionar como un predicado capaz de verdad o falsedad, «involves patiently construing each propositional context as it comes along» ${ }^{52}$. En cambio, la versión fast-track «would make sufficient remarks about truth to suggest that we need a comparable notion to regulate evaluative discourse (even though this in nonrepresentational) and then say that our adherence to propositional forms need no further explanation than that ${ }^{53}$.

Así, mientras el «[s]low track quasi-realism [...] doesn't attempt to earn the right to realist talk all in one fell swoop (via establishing the legitimacy of the truth predicate), but rather seeks to demonstrate the acceptability of different types of apparently realist talk in a more piecemeal fashion», el «[f]ast track quasi-realism begins by earning the right for the truth predicate to be applied to moral sentences, and then observes that once the legitimacy of the truth predicate is established much (all?) other realist talk comes along for free» ${ }^{54}$.

En este trabajo, dada la combinación propuesta entre escepticismo y convencionalismo, la versión de cuasirrealismo privilegiada es la denominada slow-track, ya que se ha demostrado indispensable llevar a cabo un trabajo de precisión respecto del contenido de los enunciados interpretativos. Además, este análisis permite distinguir entre enunciados interpretativos, cuyo resultado forma luego parte de la base de la decisión, y aquellos enunciados cuyo resultado no lo hace. Es decir, entre enunciados interpretativos a través de los cuáles se obtiene una norma, incluida en un enunciado de deber que es base de la decisión, de aquellos enunciados interpretativos que no desempeñan esa función. Tratándose de enunciados interpretativos cuyo resultado no es usado como base de la decisión, tanto el enunciado interpretativo como el enunciado de deber son considerados todavía susceptibles de revisión. Mientras que, en cambio, cuando el enunciado de deber es usado como base de la decisión, tanto el enunciado interpretativo como el enunciado de deber forman parte de aquellos enunciados cuya revisión no es considerada posible.

En este sentido, cuando un juez afirma que existe un deber, expresa también su actitud hacia la convención que ha usado para identificar el significado de la disposición. Así, un enunciado interpretativo consiste en la afirmación, por parte del juez, de que el significado que asocia al texto es la mejor opción dadas las convenciones interpretativas existentes. Cuando las convenciones existentes ofrecen un único significado, existe convergencia acerca del resultado interpretativo. Otras veces, la tarea se vuelve más compleja porque las convenciones interpretativas no determinan un único significado, y es en estos casos en que la actitud favorable del juez se hace evidente. Sin embargo tal actitud está presente en todos los casos.

\footnotetext{
51 BlaCKBURN, 1993: 184-186.

52 BLACKBURN, 1993: 185.

53 BLACKBURN, 1993: 185.

54 JOYCE, 2007.
} 


\section{CONSIDERACIONES FINALES}

Si la combinación del escepticismo con ambas estrategias (convencionalista y cuasirrealista) es, tal como he intentado mostrar, una empresa plausible, entonces parece posible evitar las consecuencias de los dos dilemas señalados.

Así, por un lado, la estrategia convencionalista hace espacio para evaluar una interpretación sobre la base de las convenciones interpretativas existentes. La evaluación consiste aquí en nada más que en determinar si la interpretación está apoyada por las bases convencionales. De este modo, la estrategia convencionalista permite escapar del dilema interno optando por el cuerno que contempla la alternativa pragmática. Todo ello mediante el abandono sí de la Tesis (ii), pero satisfaciendo el constraint hermenéutico y dando fuerza a la Tesis (i), en cuanto muestra cómo el uso de los métodos interpretativos, la dogmática jurídica y los sentimientos de justicia permiten explicar la atribución de significado a los textos normativos y el discurso descriptivo alrededor de esa actividad.

Por otro lado, la estrategia cuasirrealista permite disolver el dilema externo, ya que el discurso de los jueces acerca de la imposición de deberes puede ser conservado sin necesidad de abandonar la Tesis metaética. Cuando un juez afirma que la norma, asociada a la disposición mediante un enunciado interpretativo, impone un deber está expresando su actitud a favor de la convención interpretativa usada para obtener el resultado interpretativo y a favor de la norma que ha sido así obtenida. Conservando no sólo la Tesis metaética, sino también la Tesis (i), respetando el constraint hermenéutico y evitando una teoría del error.

Finalmente, antes de concluir, quisiera brevemente referirme a aquellas objeciones, dirigidas contra el cuasirrealismo, que pretenden mostrar que esta posición es indistinguible de la teoría del error. Claramente si ello fuera así, mi intento por disolver el segundo dilema fracasaría. Según el argumento que me preocupa, si se acepta que quienes profieren enunciados jurídicos tienen la intención de llevar a cabo un acto lingüístico descriptivo, entonces el cuasirrealismo parecería comprometido con afirmar que el acto efectivamente realizado no coincide con el que los hablantes tenían la intención de realizar. En otras palabras, los hablantes se equivocan acerca del acto que realizan; ello en cuanto, tal como afirma el cuasirrealismo, mediante tales actos se expresan las propias actitudes y no se describe ninguna propiedad del mundo. Esta última conclusión haría del cuasirrealismo una variante de la teoría del error ${ }^{55}$. Sin embargo, esta objeción parece asumir lo que debe probar. Es decir, dado que la forma gramatical de un enunciado (indicativo vs. imperativo) no determina qué intención posee el hablante al usarlo (informar vs. guiar el comportamiento); cuál sea la intención del hablante depende del conjunto de sus demás estados mentales y del comportamiento posterior y anterior a la emisión del enunciado. Precisamente ésta es la empresa que propone llevar a cabo el cuasirrealismo. Es decir, la de ofrecer un modo de acomodar toda esa información para atribuir al hablante una intención de formular un acto

55 Creo que este es un modo legítimo de traducir a los términos de este trabajo la objeción al expresivismo que formula CunEo (CUNEO, 2006). 
lingüístico cuasi-descriptivo. Asumir que la intención es descriptiva, tal como lo hace quien avanza esta objeción, significaría dar por descontado lo que hay que probar. El cuasirrealismo ofrece un modo de atribuir una intención, a los sujetos involucrados en la actividad interpretativa, que no implica afirmar que tienen una intención equivocada. Y en este sentido se distingue de una teoría del error.

\section{BIBLIOGRAFÍA}

ArENA, F., 2013: «Persiguiendo la tesis convencionalista», Eunomía, 5: 50-74.

BARBERIS, M., 2001: «Lo scetticismo immaginario. Nove obiezioni agli scettici à la génoise», Analisi e diritto, 2000: 1-36.

BAYÓn MoHInO, J. C., 2002: «Derecho, convencionalismo y controversia». en P. E. NAVARRO y M. C. REDONDO (eds.), La relevancia del derecho, Barcelona: Gedisa.

BlackBuRn, S., 1984: Spreading the Word: Groundings in the Philosophy of Language, Oxford: Clarendon Press.

- 1993: Essays in Quasi-Realism, New York-Oxford: Oxford UP.

- 1998: Ruling Passions, Oxford: Clarendon Press.

- 2005: «Quasi-realism No Fictionalism», en M. E. KALDERON (ed.), Fictionalism in Metaphysics, Oxford: Clarendon Press.

Bouvier, H., 2012: Particularismo y derecho. Un abordaje pospositivista en el ámbito práctico, Madrid: Marcial Pons.

Bulygin, E., 2006: El positivismo jurídico, Mexico: Fontamara.

Burge, T., 1975: «On Knowledge and Convention», The Philosophical Review, 84 (2): 249-255.

Celano, B., 2010: Fatti istituzionali, consuetudini, convenzioni, Roma: Aracne.

- 2010 [1995]: «Consuetudini, convenzioni», en B. CELANO, Fatti istituzionali, consuetudini, convenzioni, Roma: Aracne.

— 2010 [1997]: «Interazione strategica e convenzione», en B. CELANO, Fatti istituzionali, consuetudini, convenzioni, Roma: Aracne.

— 2010 [2003]: «La regola di riconoscimento è una convenzione?», en B. CELANO, Fatti istituzionali, consuetudini, convenzioni, Roma: Aracne.

Chiassoni, P., 1998: «L'ineluttabile scetticismo della "scuola genovese"», Analisi e diritto, 1998.

- 2004: «Codici interpretativi: Progetto di voce per un Vademecum giuridico», Analisi e diritto, 2002-2003: 55-124.

- 2011: Técnicas de interpretación jurídica. Brevario para juristas, Madrid: Marcial Pons.

CuneO, T., 2006: «Saying what we Mean», en R. Shafer-Landau (ed.), Oxford Studies in Metaethics, vol. 1, Oxford: Clarendon Press.

Forster, M. N., 2004: Wittgenstein on the Arbitrariness of Grammar, Princeton: Princeton University Press.

GiLberT, M., 1983: «Notes on the Concept of a Social Convention», New Literary History, 14 (2): $225-251$.

- 1993: «Is an Agreement an Exchange od Promises?», The Journal of Philosophy, 90 (12): 627-649.

- 2008: «Social Convention Revisited», Topoi, 27 (1/2): 5-16.

GuASTINI, R., 2008: Nuovi studi sull'interpretazione, Roma: Aracne. 
- 2011: Interpretare e argomentare, Milán: Giuffrè.

— 2012: «El escepticismo ante las reglas replanteado», Discusiones, 11: 27-58.

Hart, H. L. A., 1994 [1961]: The Concept of Law, en P. A. Bulloch y J. Raz (eds.), 2. a ed., Oxford: Clarendon Press.

Joyce, R., 2007: «Moral Anti-realism», Stanford Encyclopedia of Philosophy.

KeKES, J., 1989: Moral tradition and Individuality, Princeton: Princeton University Press.

LEWIS, D., 1969: Convention. A Philosophical Study, Oxford: Basil Blackwell.

MackIE, J. L., 1977: Ethics. Inventing Right and Wrong, London: Penguin (reimpresión 1990).

Marmor, A., 2009: Social Conventions. From Language to Law, Princeton: Princeton University Press.

RaWLs, J., 1971: A Theory of Justice, Cambridge: Harvard University Press.

RAz, J., 1984: «On the Nature of Rights», Mind, 93 (370): 194-214.

Ross, A., 1946 [1934]: Towards a Realistic Jurisprudence, Copenhagen: Ejnar Munksgaard.

- 1958: On Law and Justice, London: Stevens and Sons.

Russell, B., 1905: «On denoting», Mind, 14: 479-493.

SCAnlon, T., 1990: «Promises and Practices», Philosophy and Public Affairs, 19 (3): 199-226.

- 1998: What We Owe to Each Other, Cambridge: Harvard University Press.

Schiavello, A., 2010: Perché obbedire il diritto. La risposta convenzionalista e i suoi limiti, Pisa: ETS

Troper, M., 2001: La théorie du droit, le droit, l'État, Paris: PUF.

Vilajosana Rubio, J. M., 2010: El derecho en acción. La dimensión social de las normas jurídicas, Madrid: Marcial Pons.

Weber, M., 1968: Economy and Society, en G. Roth y C. Wittich (eds.), New York: Bedminster Press.

Winch, P., 2008 [1990, 1958]: The Idea of a Social Science and its Relation to Philosophy, Abingdon-New York: Routledge. 